\title{
High-order-harmonic generation from molecular isomers with midinfrared intense laser pulses
}

\author{
Anh-Thu Le, ${ }^{1}$ R. R. Lucchese, ${ }^{2}$ and C. D. Lin $^{1}$ \\ ${ }^{1}$ Department of Physics, Cardwell Hall, Kansas State University, Manhattan, Kansas 66506, USA \\ ${ }^{2}$ Department of Chemistry, Texas A\&M University, College Station, Texas 77843-3255, USA
}

(Received 13 May 2013; published 12 August 2013)

\begin{abstract}
We present theoretical calculations of high-order-harmonic generation (HHG) from stereoisomers of 1,2dichloroethylene $\left(\mathrm{C}_{2} \mathrm{H}_{2} \mathrm{Cl}_{2}\right)$ and 2-butene $\left(\mathrm{C}_{4} \mathrm{H}_{8}\right)$ based on the quantitative rescattering theory. Our results show that the HHG spectra from these cis and trans isomers with intense midinfrared laser pulses are clearly distinguishable, even when the molecules are randomly oriented, in good agreement with the recent experiments by Wong et al. [Phys. Rev. A 84, 051403(R) (2011)]. We found that the angle-averaged tunneling ionization yields and photoionization cross sections from the cis and trans isomers for both molecules are nearly identical. The origin of the differences in HHG spectra is traced as due to the interplay in the angular dependence of the photoionization (or photorecombination) cross section and ionization rate.
\end{abstract}

DOI: 10.1103/PhysRevA.88.021402

PACS number(s): $33.80 . \mathrm{Wz}, 33.80 . \mathrm{Eh}, 42.65 . \mathrm{Ky}$

High-order-harmonic generation (HHG) has been shown both experimentally and theoretically to contain information about the target molecular structure and dynamics, which are encoded in the amplitude and phase of the emitted high-order harmonics [1-12]. HHG spectroscopy for simple molecules has been greatly benefited from the ability to align molecules [13]. In fact, rich information contained in HHG spectra from aligned molecules is mostly washed out if the molecules are not well aligned. Whereas earlier studies have focused mostly on simple molecules with the use of infrared lasers, more recent studies in HHG spectroscopy have now expanded to more complex targets and by using midinfrared lasers [14-17].

As the geometric arrangement of atoms in stereoisomers differs, they can serve as interesting tests for HHG spectroscopy. In a recent paper by Wong et al. [16], HHG spectra from stereoisomers of 1,2-dichloroethylene (1,2-DCE, $\left.\mathrm{C}_{2} \mathrm{H}_{2} \mathrm{Cl}_{2}\right)$ and 2-butene $\left(\mathrm{C}_{4} \mathrm{H}_{8}\right)$ have been reported. It was found that the spectra from these cis and trans isomers are quite distinguishable in a broad range of energy, even when the molecules are not aligned. The mechanism behind this was attributed as due to the differences in the strong-field ionization. The ability to distinguish nonaligned isomers by HHG spectroscopy is of great interest. In particular, it opens up the possibility to study fast structural changes during the isomerization process using HHG [18] even without the need of molecular alignment.

In this Rapid Communication we employ the quantitative rescattering $(\mathrm{QRS})$ theory $[4,19,20]$ as extended to polyatomic targets [21] to calculate HHG spectra from cis and trans stereoisomers of 1,2-DCE and 2-butene under intense midinfrared laser pulses. Our calculation shows good agreements with experiment by Wong et al. [16]. However, we found that the angle-averaged ionization yields from $c i$ s and trans isomers are nearly indistinguishable. Here we provide an analysis of the nature of the differences in HHG spectra from these isomers.

Within the QRS, the laser-induced dipole $D(\omega, \theta, \phi)$ for a molecule in a linearly polarized intense laser pulse can be written as a product of a returning electron wave packet $W(E, \theta, \phi)$ and a photorecombination (time inverse of photoionization) transition dipole $d(\omega, \theta, \phi)[19,20]$,

$$
D(\omega, \theta, \phi)=W(E, \theta, \phi) d(\omega, \theta, \phi),
$$

where $\omega$ is the photon energy of the emitted harmonic, and $\theta$ and $\phi$ are the polar and azimuthal angles, describing the direction of the laser polarization with respect to the $Z$ axis of the molecular frame (see the insets of Fig. 1). Here, electron energy $E$ is related to the emitted photon energy $\omega$ by $E=$ $\omega-I_{p}$, with $I_{p}$ being the ionization potential of the target. We follow a version of the QRS in which a reference atom is used to calculate the energy-dependent returning electron wave packet, with the additional (alignment-dependent) phase $\Delta \eta(\theta, \phi)$ approximated by the phase of the asymptotic initial wave function of the active electron [21],

$$
D(\omega, \theta, \phi)=W^{\mathrm{ref}}(\omega) N^{1 / 2}(\theta, \phi) e^{i \Delta \eta(\theta, \phi)} d(\omega, \theta, \phi) .
$$

For the case of nonaligned molecules, Eq. (2) needs to be averaged over the isotropic molecular distribution [19,21].

A major advantage of this approach over the more standard approach of calculating the electron wave packet directly for the target using the strong-field approximation (SFA) is that it avoids possible spurious spikes, which occur quite frequently for polyatomic targets. For a reference atom one can choose the ground state of a scaled hydrogenlike atom, in which the nuclear charge is chosen to have the same ionization potential as the target [1,4,19,22-24]. According to the QRS, an overall (energy-independent) magnitude of the electron wave packet $W(E, \theta, \phi)$ is proportional to the (alignment-dependent) ionization amplitude $N^{1 / 2}(\theta, \phi)$ for electron emission along the laser polarization, which can be calculated by using the SFA or the molecular tunneling ionization [molecular orbital Ammosov-Delone-Krainov (MO-ADK)] theory [25]. Molecular photoionization has been studied extensively over the past five decades with various theoretical tools to calculate the photoionization cross sections. Here we use the ePolyScat package [26] for the transition dipole of polyatomic molecules. We treat the active electron as represented by the highest occupied molecular orbital (HOMO). The HOMO wave functions are calculated using the Gaussian quantum chemistry code [27]. We typically use the augmented correlation-consistent polarized valence triple-zeta (aug-cc-pVTZ) basis set at the Hartree-Fock level. In the following we will first consider the case of $\mathrm{C}_{2} \mathrm{H}_{2} \mathrm{Cl}_{2}$. 


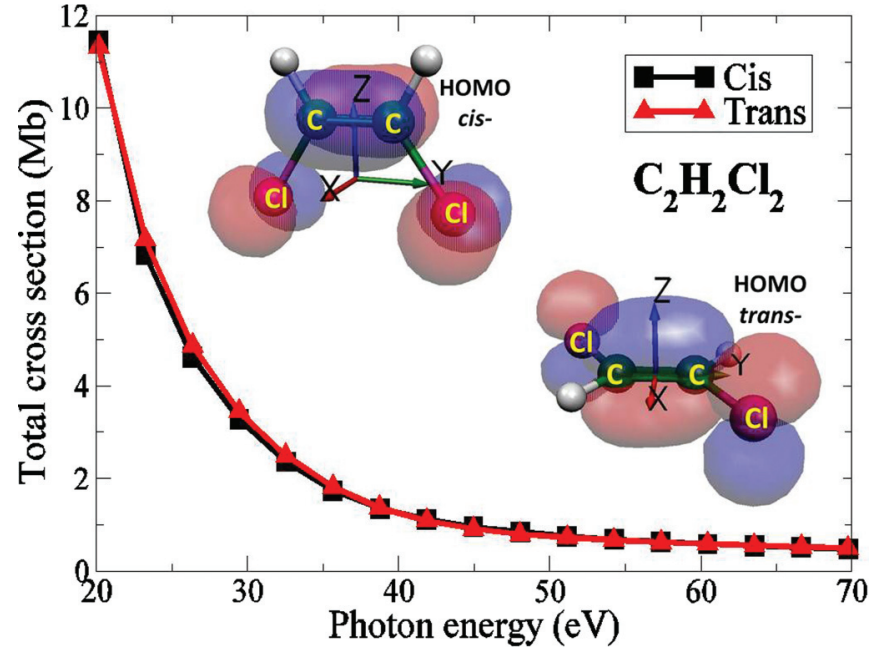

FIG. 1. (Color online) Total (integrated) photoionization cross section from the HOMO of cis-DCE and trans-DCE. The insets show the HOMOs and molecular frame coordinates used in our calculations. All atoms are in the $Y Z$ plane for cis-DCE and the $X Y$ plane for trans-DCE.

The ePolyScat results for photoionization cross sections from the HOMO of cis- and trans-DCE are shown in Fig. 1 for the case of nonaligned molecules. The cross sections are nearly identical in the range of energy below $70 \mathrm{eV}$. Since their ionization potentials are also nearly identical $(\approx 9.6 \mathrm{eV})$, one might speculate that the differences in spectra observed by Wong et al. [16] would come from the differences in ionization rates. In fact, that was the original explanation by Wong et al. [16]. Our calculations using the MO-ADK, however, showed that the ionization rates from the HOMOs for the two isomers are very close to each other over a typical intensity range from $0.5 \times 10^{14}$ to $1.5 \times 10^{14} \mathrm{~W} / \mathrm{cm}^{2}$, with the largest difference being only about $30 \%$. Within the SFA with different laser wavelengths of $800,1300,1500$, and $1800 \mathrm{~nm}$ in a similar intensity range, the ionization rates from the two isomers agree to within about $10 \%$. In both methods, ionization from trans-DCE is slightly stronger than cis-DCE. As ionization and photorecombination are two major steps in the HHG process, it is not quite obvious how to reconcile the observed differences in HHG spectra from the two isomers with similarities found in their ionization rates and photoionization cross sections. We will show below that the main features in the experimental data by Wong et al. [16] are reproducible by the QRS. We note that our ionization rates are in disagreement with theoretical results by Wong et al. [16], who found that the ionization from cis-DCE is much stronger than from trans-DCE. Although no real HHG calculation was reported in Wong et al. [16], they attributed the stronger HHG yield from $c i s$-DCE as mainly due to its stronger ionization.

Before presenting the HHG spectra from the QRS, we analyze the alignment-dependent ionization from the two isomers. We show in Figs. 2(a) and 2(b) the ionization rates for the HOMO of cis-DCE and trans-DCE versus the laser polarization direction $\{\theta, \phi\}$, which is defined with respect to the molecular frame (see the insets of Fig. 1). The azimuthal angle $\phi$ is measured from the $x$ axis. The calculations were carried out using the MO-ADK $[25,28]$ at a laser intensity of
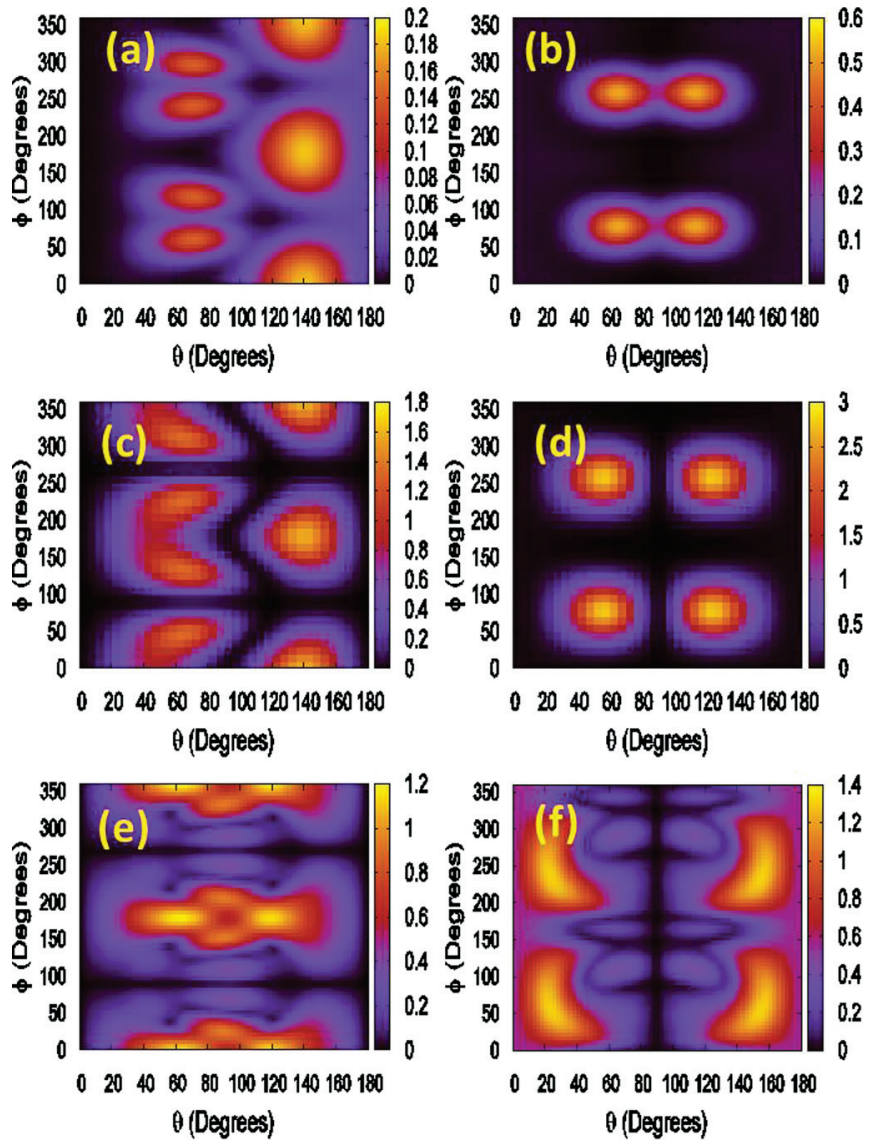

FIG. 2. (Color online) (a), (b) Ionization rate from MO-ADK for cis-DCE and trans-DCE, respectively, at an intensity of $0.6 \times$ $10^{14} \mathrm{~W} / \mathrm{cm}^{2}$. (c), (d) Ionization probability (scaled by a factor of 100 ) from the SFA for the HOMO of cis-DCE and trans-DCE, respectively. Only the electron emission along the laser polarization is included during a half-cycle laser pulse of a $1800 \mathrm{~nm}$ wavelength and an intensity of $0.6 \times 10^{14} \mathrm{~W} / \mathrm{cm}^{2}$. (e), (f) Transition dipole amplitude for cis-DCE and trans-DCE, respectively, at a photon energy of $39 \mathrm{eV}$.

$0.6 \times 10^{14} \mathrm{~W} / \mathrm{cm}^{2}$. The angular dependence is very different for the two isomers. For cis-DCE, the ionization yield is stronger when the field direction is closer to $\theta=180^{\circ}$, i.e., pointed away from the $\mathrm{C}-\mathrm{C}$ center (or the electric force is closer to $\theta=0^{\circ}$, or pointed toward the $\mathrm{C}-\mathrm{C}$ center). We comment that the angular dependence of the ionization rate looks very similar to that of the asymptotic wave function of the HOMO [21,28]. Overall, our angular dependence resembles the results of Spanner and Patchkovskii [29]. However, the angle-averaged rate (for nonaligned molecules) for trans-DCE is about $30 \%$ stronger than that of cis-DCE. We found that the relative differences between the ionization rates from the two isomers remain nearly the same for the intensity range from $0.5 \times 10^{14}$ to $1.5 \times 10^{14} \mathrm{~W} / \mathrm{cm}^{2}$.

We also carried out calculations for ionization yields using the SFA. Results are presented in Figs. 2(c) and 2(d) for cis-DCE and trans-DCE for a half-cycle yield with a laser of $1800 \mathrm{~nm}$ wavelength and an intensity of $0.6 \times 10^{14} \mathrm{~W} / \mathrm{cm}^{2}$. Here, only electron emission along the laser polarization direction is included. The results look quite similar to that of 
the MO-ADK, shown in Figs. 2(a) and 2(b). However, for cisDCE, the yield is slightly stronger when the laser polarization is pointed toward the C-C center. The angle-averaged yield for trans-DCE is about $10 \%$ higher than that of cis-DCE. Although emission should be dominant along the laser polarization in the tunneling regime, we comment that the total, integrated over all emission directions, yields for both isomers (not shown) are much closer to that of the MO-ADK, shown in Figs. 2(a) and 2(b). In particular, the alignment dependence of the total yield near $\theta=30^{\circ}-90^{\circ}$ for $c i s$-DCE is nearly identical to that of MO-ADK shown in Fig. 2(a). Nevertheless, the total yield is still slightly more preferable when the laser polarization is pointed toward the C-C center. For trans-DCE, the total yield becomes sharper as a function of $\phi$ near $\phi=80^{\circ}$ and $280^{\circ}$, i.e., it is also much closer to the MO-ADK result shown in Fig. 2(b). We should point out that, strictly speaking, only the electron emitted along the laser polarization direction is relevant to the HHG process, since it can return to the parent ion. The SFA yields for the two isomers are found to agree to within about $10 \%$ for different wavelengths in the intensity range from $0.5 \times 10^{14}$ to $1.5 \times 10^{14} \mathrm{~W} / \mathrm{cm}^{2}$.

For completeness, we show in Figs. 2(e) and 2(f) a comparison of the transition dipole amplitude from the ePolyScat package [26] for cis-DCE and trans-DCE, respectively, at a photon energy of $39 \mathrm{eV}$. We note that, although the overall magnitude of the transition dipoles for the two isomers is quite close, their angular dependence is very different. In particular, the transition dipole for trans-DCE at $39 \mathrm{eV}$ peaks near $\theta \leqslant 40^{\circ}$ and $\theta \geqslant 140^{\circ}$, where the ionization is insignificant. This would potentially reduce the HHG yields from trans-DCE.

We now compare in Fig. 3(a) the HHG spectra from cisDCE and trans-DCE. The calculations were carried out using the QRS with a $1800 \mathrm{~nm}$ wavelength laser pulse of an intensity of $0.6 \times 10^{14} \mathrm{~W} / \mathrm{cm}^{2}$ and $40 \mathrm{fs}$ duration. The ionization rates from the SFA were used. Note that we have adjusted the laser intensity as compared to the estimate of $1.1 \times 10^{14} \mathrm{~W} / \mathrm{cm}^{2}$ in Wong et al. [16] to match the experimental cutoff positions. A striking feature is the presence of a strong minimum near $43 \mathrm{eV}$ in the spectrum from trans-DCE. This "Cooper-type" minimum is in good agreement with the experiment by Wong et al. [16], who found a deep minimum near $42 \mathrm{eV}$. The overall shape of both spectra is also in relatively good agreement with Wong et al. [16]. Our results disagree with experiment for energies below about $30 \mathrm{eV}$, where the experimental yields drop quickly for both isomers [16]. The discrepancy could be due to the neglect of the macroscopic propagation effect. In particular, the absorption of HHG photons is known to reduce the HHG yield more strongly at lower photon energies (see, for example, Ref. [30]). We further note that the shallow minimum slightly below $40 \mathrm{eV}$ which was observed by Wong et al. [16] for cis-DCE [see their Fig. 1(a)] was not reproduced by the QRS.

We show in Fig. 3(b) the QRS intensity ratio between cisDCE and trans-DCE for different laser wavelengths of 1300, 1500 , and $1800 \mathrm{~nm}$, at a laser intensity of $0.6 \times 10^{14} \mathrm{~W} / \mathrm{cm}^{2}$. For the case of $1800 \mathrm{~nm}$, our intensity ratio agrees quite well with Wong et al. [16] for energies above $30 \mathrm{eV}$. In particular,
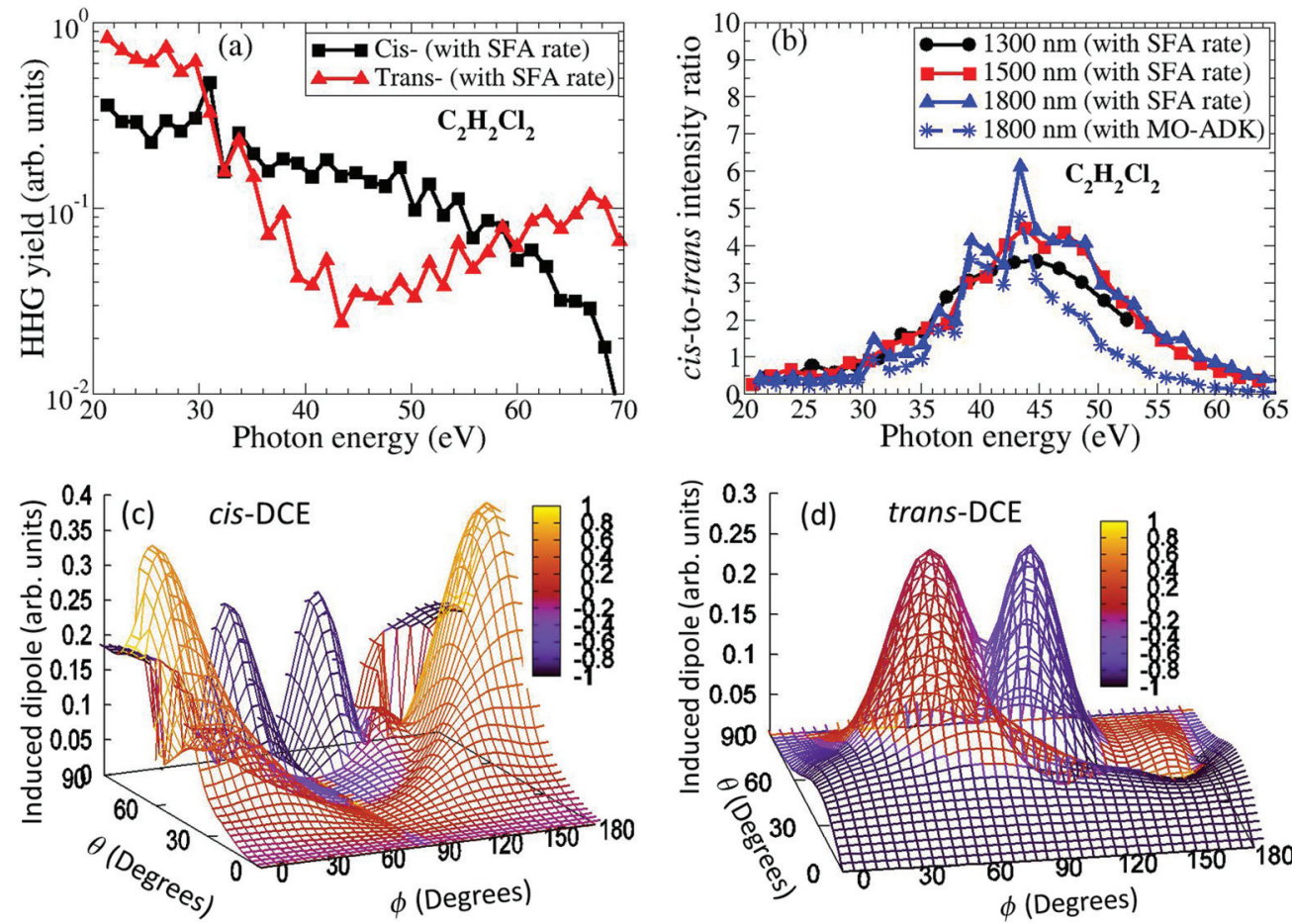

FIG. 3. (Color online) (a) HHG spectra (only the envelopes are shown) from cis- and trans-DCE for a 1800 nm wavelength laser and an intensity of $0.6 \times 10^{14} \mathrm{~W} / \mathrm{cm}^{2}$. The calculations were carried out with the QRS using the SFA ionization rate. (b) cis-DCE to trans-DCE intensity ratio for different laser wavelengths of 1300, 1500, and $1800 \mathrm{~nm}$. The laser intensity is fixed at $0.6 \times 10^{14} \mathrm{~W} / \mathrm{cm}^{2}$. (c) Induced dipole amplitude (weighted by $\sin \theta$, vertical axis) and phase (color code) for $\mathrm{H} 65$ (photon energy of $45 \mathrm{eV}$ ) from cis-DCE. The phase is given in units of $\pi$. (d) Same as (c) but for trans-DCE. The laser parameters are the same as for (a). 

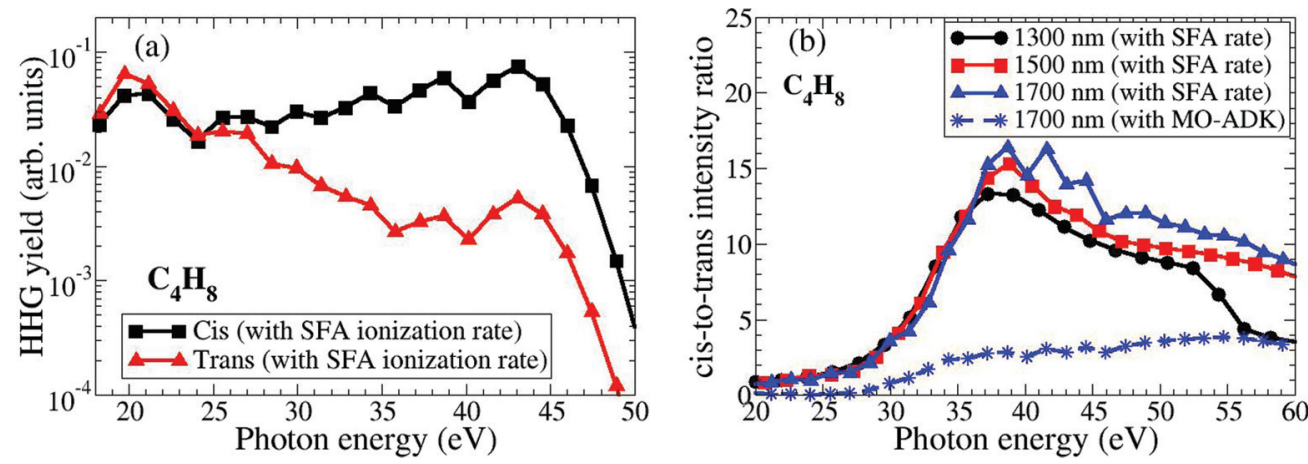

FIG. 4. (Color online) (a) HHG spectra (only the envelopes are shown) from cis and trans isomers of $\mathrm{C}_{4} \mathrm{H}_{8}$ for a $1700 \mathrm{~nm}$ wavelength laser with an intensity of $0.4 \times 10^{14} \mathrm{~W} / \mathrm{cm}^{2}$, and a pulse duration of $40 \mathrm{fs}$. The calculations were carried out with the QRS using the SFA ionization rate. (b) cis to trans intensity ratio for different laser wavelengths of 1300,1500 , and $1700 \mathrm{~nm}$. The laser intensity is fixed at $0.6 \times 10^{14} \mathrm{~W} / \mathrm{cm}^{2}$. The QRS result with the use of the MO-ADK rate is also shown for the case of $1700 \mathrm{~nm}$.

a strong peak is seen near $43 \mathrm{eV}$, compared to near $40 \mathrm{eV}$ by Wong et al. [16]. Clearly, this peak is associated with the Cooper-type minimum in the spectrum from trans-DCE, since the spectrum from the cis-DCE is rather monotonic in this energy range. For comparison we also show the QRS result with the use of the MO-ADK ionization rate (dashed line). The theoretical intensity ratio is only slightly reduced with the laser wavelength. On the contrary, the ratio observed by Wong et al. [16] changes quite significantly with laser wavelength. At present the nature of this strong wavelength dependence is not understood. On the other hand, both experiment and theory show that the position of the ratio peak changes very slightly with laser wavelength and intensity. Within the QRS, this stability is not surprising. It reflects the fact that the Cooper-type minimum is due to the target structure and is largely laser independent.

As noted above, the overlap in the angular dependence of the ionization rate and transition dipole is quite small for trans-DCE, which could result in small HHG yields. To further understand the nature of the Cooper-type minimum in trans-DCE, we compare in Figs. 3(c) and 3(d) the amplitude and phase (color code) of the induced dipole $D(\omega, \theta, \phi)$ for H65 (photon energy $\omega=45 \mathrm{eV}$ ) for cis-DCE and trans-DCE, respectively. We note quite a strong destructive interference due to different molecular alignments in trans-DCE, whereas the contributions from different alignments are mostly in phase for cis-DCE. Furthermore, according to Eq. (2), apart from an overall alignment-independent factor [the wave packet for a reference atom $W^{\text {ref }}(\omega)$ ], the only laser-dependent factor is the ionization rate $N(\theta, \phi)$. As discussed above, the alignment dependence of the tunneling ionization is only weakly changed with laser parameters. The QRS therefore predicts the stability of this destructive (or constructive) interference in trans-DCE (or $c i s$-DCE). This results in the stability of the Cooper-type minimum in trans-DCE. We comment that the Cooper-type minimum could also have a different nature due to a minimum in the transition dipole amplitude as a function of energy, as has been discussed before, for example, in the case of $\mathrm{CCl}_{4}[17,21]$. For 1,2-DCE, there is no obvious minimum in the photoionization cross section (see Fig. 1).

Similar calculations were carried out for cis- and trans2-butene. Again, both SFA and MO-ADK calculations show that the ionization rates from the two isomers are quite close. Typical spectra from the QRS using the SFA ionization rates are shown in Fig. 4(a) for a $1700 \mathrm{~nm}$ wavelength laser pulse with an intensity of $0.4 \times 10^{14} \mathrm{~W} / \mathrm{cm}^{2}$. The laser intensity was adjusted to match the cutoff position with the experiment. The shapes of the spectra are in relatively good agreement with Wong et al. [16]. However, the cis to trans intensity ratio reaches a value of 16 , as shown in Fig. 4(b), which is about four times larger than the experimental value [16]. The QRS result obtained with the MO-ADK rate is also shown for the case of a laser wavelength of $1700 \mathrm{~nm}$ (dashed line), which seems to agree better with the experiment by Wong et al. [16].

In conclusion, we have presented calculations based on the QRS for HHG from randomly oriented stereoisomers of 1,2-DCE and 2-butene in intense midinfrared laser pulses. While the results are quite encouraging for 1,2-DCE, there remain some discrepancies with experiments by Wong et al. [16], especially for 2-butene. Although the stability of the position of the Cooper-type minimum in trans-DCE can be explained within the QRS approach, the sensitivity of the intensity ratio with respect to the laser parameters is not understood at present. There are still some limitations in our simulations. First, we have not included the ground-state depletion, which should be relevant for the laser parameters used in Wong et al. [16]. This would add an extra angular dependence to the induced dipole $D(\omega, \theta, \phi)$. Second, the treatment of ionization for polar molecules might need to go beyond the standard MO-ADK or SFA [31-38]. Lastly, the macroscopic propagation needs to be included for a realistic comparison with experiments. Future experiments, especially with aligned isomers, are extremely desirable, which would provide more detailed information to help validate different aspects of theory as well as to identify the most important physics to be accounted for in future simulations.

We thank V. R. Bhardwaj for stimulating discussions. This work was supported in part by the Chemical Sciences, Geosciences and Biosciences Division, Office of Basic Energy Sciences, Office of Science, US Department of Energy. 
[1] J. Itatani, J. Levesque, D. Zeidler, H. Niikura, H. Pepen, J. C. Kiefer, P. B. Corkum, and D. M. Villeneuve, Nature (London) 432, 867 (2004).

[2] S. Baker, J. S. Robinson, C. A. Haworth, H. Teng, R. A. Smith, C. C. Chirila, M. Lein, J. W. G. Tisch, and J. P. Marangos, Science 312, 424 (2006).

[3] M. Lein, J. Phys. B 40, R135 (2007).

[4] T. Morishita, A. T. Le, Z. Chen, and C. D. Lin, Phys. Rev. Lett. 100, 013903 (2008).

[5] A. T. Le, R. R. Lucchese, M. T. Lee, and C. D. Lin, Phys. Rev. Lett. 102, 203001 (2009).

[6] M. V. Frolov, N. L. Manakov, T. S. Sarantseva, and A. F. Starace, Phys. Rev. A 83, 043416 (2011).

[7] S. Minemoto, T. Umegaki, Y. Oguchi, T. Morishita, A. T. Le, S. Watanabe, and H. Sakai, Phys. Rev. A 78, 061402(R) (2008).

[8] H. J. Wörner, H. Niikura, J. B. Bertrand, P. B. Corkum, and D. M. Villeneuve, Phys. Rev. Lett. 102, 103901 (2009).

[9] W. Boutu, S. Haessler, H. Merdji, P. Breger, G. Waters, M. Stankiewicz, L. J. Frasinski, R. Taieb, J. Caillat, A. Maquet, P. Monchicourt, B. Carre, and P. Salieres, Nat. Phys. 4, 545 (2008).

[10] O. Smirnova, Y. Mairesse, S. Patchkovskii, N. Dudovich, D. Villeneuve, P. Corkum, and M. Yu. Ivanov, Nature (London) 460, 972 (2009).

[11] S. Haessler, J. Caillat, W. Boutu, C. Giovanetti-Teixeira, T. Ruchon, T. Auguste, Z. Diveki, P. Breger, A. Maquet, B. Carre, R. Taieb, and P. Salieres, Nat. Phys. 6, 200 (2010).

[12] C. Vozzi, M. Negro, F. Calegari, G. Sansone, M. Nisoli, S. De Silvestri, and S. Stagira, Nat. Phys. 7, 822 (2011).

[13] H. Stapelfeldt and T. Seideman, Rev. Mod. Phys. 75, 543 (2003).

[14] R. Torres, N. Kajumba, J. G. Underwood, J. S. Robinson, S. Baker, J. W. G. Tisch, R. de Nalda, W. A. Bryan, R. Velotta, C. Altucci, I. C. E. Turcu, and J. P. Marangos, Phys. Rev. Lett. 98, 203007 (2007).

[15] M. C. H. Wong, J.-P. Brichta, and V. R. Bhardwaj, Phys. Rev. A 81, 061402(R) (2010).

[16] M. C. H. Wong, J.-P. Brichta, M. Spanner, S. Patchkovskii, and V. R. Bhardwaj, Phys. Rev. A 84, 051403 (2011).

[17] M. C. H. Wong, A. T. Le, A. F. Alharbi, A. E. Boguslavskiy, R. R. Lucchese, J.-P. Brichta, C. D. Lin, and V. R. Bhardwaj, Phys. Rev. Lett. 110, 033006 (2013).

[18] V. H. Le, A. T. Le, R. H. Xie, and C. D. Lin, Phys. Rev. A 76, 013414 (2007).
[19] A. T. Le, R. R. Lucchese, S. Tonzani, T. Morishita, and C. D. Lin, Phys. Rev. A 80, 013401 (2009).

[20] C. D. Lin, A. T. Le, Z. Chen, T. Morishita, and R. R. Lucchese, J. Phys. B 43, 122001 (2010).

[21] A. T. Le, R. R. Lucchese, and C. D. Lin, Phys. Rev. A 87, 063406 (2013).

[22] J. Levesque, D. Zeidler, J. P. Marangos, P. B. Corkum, and D. M. Villeneuve, Phys. Rev. Lett. 98, 183903 (2007).

[23] A. T. Le, T. Morishita, and C. D. Lin, Phys. Rev. A 78, 023814 (2008).

[24] A. T. Le, R. D. Picca, P. D. Fainstein, D. A. Telnov, M. Lein, and C. D. Lin, J. Phys. B 41, 081002 (2008).

[25] X. M. Tong, Z. X. Zhao, and C. D. Lin, Phys. Rev. A 66, 033402 (2002).

[26] A. P. P. Natalense and R. R. Lucchese, J. Chem. Phys. 111, 5344 (1999).

[27] M. J. Frisch et al., GAUSSIAN 03, revision C.02 (Gaussian Inc., Pittsburgh, PA, 2003).

[28] S. F. Zhao, J. Xu, C. Jin, A. T. Le, and C. D. Lin, J. Phys. B 44, 035601 (2011).

[29] M. Spanner and S. Patchkovskii, Chem. Phys. 414, 10 (2013).

[30] G. Wang, C. Jin, A. T. Le, and C. D. Lin, Phys. Rev. A 84, 053404 (2011).

[31] A. Etches and L. B. Madsen, J. Phys. B 43, 155602 (2010).

[32] M. Abu-samha and L. B. Madsen, Phys. Rev. A 82, 043413 (2010).

[33] O. I. Tolstikhin, T. Morishita, and L. B. Madsen, Phys. Rev. A 84, 053423 (2011).

[34] L. B. Madsen, O. I. Tolstikhin, and T. Morishita, Phys. Rev. A 85, 053404 (2012).

[35] L. B. Madsen, F. Jensen, O. I. Tolstikhin, and T. Morishita, Phys. Rev. A 87, 013406 (2013).

[36] L. Holmegaard, J. L. Hansen, L. Kalhoj, S. L. Kragh, H. Stapelfeldt, F. Filsinger, J. Küpper, G. Meijer, D. Dimitrovski, M. Abu-samha, C. P. J. Martiny, and L. B. Madsen, Nat. Phys. 6, 428 (2010).

[37] H. Li, D. Ray, S. De, I. Znakovskaya, W. Cao, G. Laurent, Z. Wang, M. F. Kling, A. T. Le, and C. L. Cocke, Phys. Rev. A 84, 043429 (2011).

[38] J. Wu, L. P. H. Schmidt, M. Kunitski, M. Meckel, S. Voss, H. Sann, H. Kim, T. Jahnke, A. Czasch, and R. Dörner, Phys. Rev. Lett. 108, 183001 (2012). 